\begin{tabular}{|c|c|}
\hline Title & $\begin{array}{l}\text { Symmetric quantum dots as efficient sources of highly entangled photons: Violation of Bell's inequality without spectral } \\
\text { and temporal filtering }\end{array}$ \\
\hline Author(s) & $\begin{array}{l}\text { Kuroda, Takashi; Mano, Takaaki; Ha, Neul; Nakaj ima, Hideaki; Kumano, Hidekazu; Urbaszek, Bernhard; Jo, } \\
\text { Masafumi; A bbarchi, Marco; Sakuma, Y oshiki; Sakoda, Kazuaki; Suemune, Ikuo; Marie, X Xvier; A mand, Thierry }\end{array}$ \\
\hline Citation & $\begin{array}{l}\text { Physical Review B, 88(4), 041306-1-041306-5 } \\
\text { https://doi.org/10.1103/PhysRevB.88.041306 }\end{array}$ \\
\hline Issue Date & $2013-07-18$ \\
\hline Doc URL & http:/hdl. handle.net/2115/59632 \\
\hline Rights & (-2013 A merican Physical Society \\
\hline Type & article \\
\hline File Information & Kuroda_PRB13.pdf \\
\hline
\end{tabular}

Instructions for use 


\title{
gु \\ Symmetric quantum dots as efficient sources of highly entangled photons: Violation of Bell's inequality without spectral and temporal filtering
}

\author{
Takashi Kuroda, ${ }^{1,2}$ Takaaki Mano, ${ }^{1}$ Neul Ha ${ }^{1,2}$ Hideaki Nakajima,${ }^{1,3}$ Hidekazu Kumano, ${ }^{3}$ Bernhard Urbaszek,,${ }^{4}$ Masafumi Jo, ${ }^{1}$ \\ Marco Abbarchi, ${ }^{1}$ Yoshiki Sakuma, ${ }^{1}$ Kazuaki Sakoda, ${ }^{1}$ Ikuo Suemune, ${ }^{3}$ Xavier Marie,${ }^{4}$ and Thierry Amand ${ }^{4}$ \\ ${ }^{1}$ National Institute for Materials Science, 1 Namiki, Tsukuba 305-0044, Japan \\ ${ }^{2}$ Graduate School of Engineering, Kyushu University, Japan \\ ${ }^{3}$ Research Institute for Electronic Science, Hokkaido University, Sapporo 001-0021, Japan \\ ${ }^{4}$ Université de Toulouse, INSA-CNRS-UPS, LPCNO, 135 avenue de Rangueil, 31077 Toulouse, France
}

(Received 26 February 2013; published 18 July 2013)

\begin{abstract}
An ideal emitter of entangled photon pairs combines the perfect symmetry of an atom with the convenient electrical trigger of light sources based on semiconductor quantum dots. Our source consists of strain-free GaAs dots self-assembled on a triangular symmetric (111)A surface. The emitted photons reveal a fidelity to the Bell state as high as $86( \pm 2) \%$ without postselection. We show a violation of Bell's inequality by more than five times the standard deviation, a prerequisite to test a quantum cryptography channel for eavesdropping. Due to the strict nonlocal nature the source can be used for real quantum processing without any postprocessing. The remaining decoherence channel of the photon source is ascribed to random charge and nuclear spin fluctuations in and near the dot.
\end{abstract}

DOI: 10.1103/PhysRevB.88.041306

PACS number(s): 78.67.Hc, 03.67.Bg, 78.55.-m

Introduction. Entanglement is an essential resource for the implementation of quantum information processing. In addition to the demonstration of quantum correlations, a more stringent criterion for the direct implementation of an entangled photon source in quantum processing is the violation of Bell's inequality, initially proposed as an experimental verification for quantum nonlocality. ${ }^{1,2}$ In the original quantum cryptography scheme of Ekert, ${ }^{3}$ Bell's inequality is used as a test of the safety (against eavesdropping) of the key distribution, as experimentally verified for sources based on parametric down-conversion. 4 ., The use of a semiconductor quantum dot as a triggered photon source was initially proposed in $2000 .{ }^{6}$ Despite the concept being straightforward and analogous to that of an atomic cascade employed in the first demonstration of the violation of Bell's theorem, ${ }^{7,8}$ experimental implementation remains challenging due to the inherent anisotropy of dots.

Most investigated dot systems suffer from structural asymmetry, which induces a fine structure splitting (FSS) of the optically active exciton states. ${ }^{9,10}$ This FSS makes radiative transition paths distinguishable, and thus strongly degrades or even prohibits entanglement in the emitted photons. ${ }^{11}$ Sophisticated techniques have been developed to recover the optical isotropy of dots, eventually demonstrating entangled photon pair emission. ${ }^{12-19}$ Despite impressive progress, these postproduction techniques suffer from two main drawbacks. First, the application of external parameters such as strain and/or electric fields has to be fine tuned specifically for each fabricated dot. Second, the degree of entanglement remains low compared with those routinely achieved with other nondeterministic sources. Importantly, a violation of Bell's inequality has only been shown up to now by selecting photons emitted during a restricted temporal window ${ }^{20}$ or with a very specific wavelength. ${ }^{14}$

Here we take a different approach to create a perfectly symmetric photon source using an alternative method of dot selfassembly, namely, droplet epitaxy on (111)A substrates. ${ }^{21-23}$
The photon pairs emitted by our source exhibit a high polarization entanglement fidelity of $f=0.86 \pm 0.02$. The nonlocal nature of the observed entanglement is quantified in additional measurements in nonorthogonal polarization bases. We show that Bell's inequality is violated as $S=$ $2.33 \pm 0.06>2$ in as-grown samples, without any need for the spectral or temporal postselection previously employed for InAs dots. ${ }^{14,20}$. This allows to use our source in principle directly in the Ekert scheme for quantum cryptography as the quantum channel is secured directly against eavesdropping by the completeness of quantum mechanics. ${ }^{24}$

Samples and setup. Symmetry breaking in conventional dots is related to the growth of a cubic semiconductor along the [100] crystal axis. Since a (100) surface has atomic $C_{2 v}$ symmetry, structures grown on it inevitably suffer from elongation, which lifts the degeneracy of the exciton state ${ }^{25}$ [see Fig. 1(a)]. In contrast, in dots grown along the [111] axis, where both (111) $A$ and (111) $B$ surfaces have $C_{3 v}$ symmetry, any source of structural asymmetry is eliminated. ${ }^{26,27}$ As a consequence, the exciton states remain degenerate. Unfortunately, the standard dot growth in the Stranski-Krastanov mode is prohibited along [111]. This obstacle is overcome by using patterned substrates ${ }^{28,29}$ or droplet epitaxy. ${ }^{21,30}$ In InGaAs dots on a patterned (111) $B$ substrate, the suppression of the FSS and classical correlations ${ }^{29}$ have been demonstrated.

We employ GaAs dots grown on a (111)A substrate by droplet epitaxy. ${ }^{21,22}$ This technique allows dots to be embedded in a lattice-matched barrier material, which ensures the robustness of the suppression of the FSS against microscopic randomness. Also, as droplet epitaxy is not strain driven, a large variety of dot-barrier material combinations can be grown. This allows in principle for tuning of the dot emission wavelength while maintaining close to zero FSS due to the high dot symmetry. This is an important advantage compared to annealed InGaAs dots, which also exhibit close to zero fine structure splitting but for a specific wavelength band. ${ }^{31}$ 
(a)

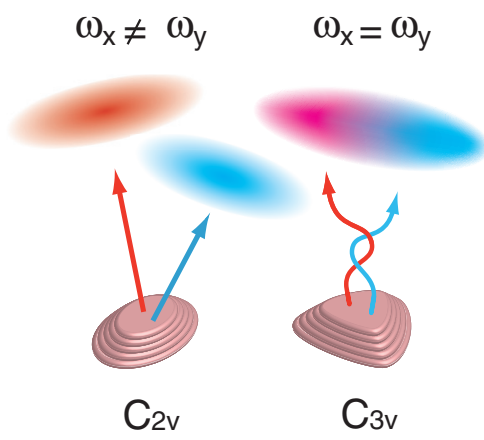

(b)
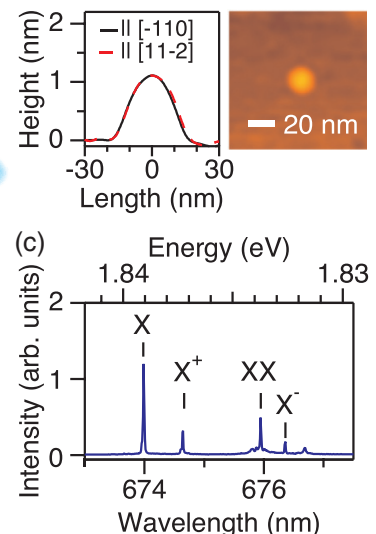

FIG. 1. (Color online) (a) Conventional dots are grown on a (100) surface that has $C_{2 v}$ symmetry. The elongation of the dot shape and other anisotropic properties induce the asymmetry of the wave function envelope. This causes the exciton state to split into two orthogonally polarized states with energies of $\omega_{x}$ and $\omega_{y}$. In contrast, for dots grown on a (111) surface that has $C_{3 v}$ symmetry, the exciton states remain degenerate. (b) Atomic force microscope analysis of the sample surface. (c) PL spectrum of an isolated GaAs dot. See text for nomenclature.

The details of dot growth are reported elsewhere. ${ }^{21} \mathrm{We}$ employed a standard molecular beam epitaxy machine. After growing an $\mathrm{Al}_{0.3} \mathrm{Ga}_{0.7} \mathrm{As}$ layer on the gallium-rich surface of a GaAs (111)A substrate, we supplied a 0.043 monolayer of gallium that formed $\mathrm{Ga}$ droplets at $400{ }^{\circ} \mathrm{C}$. Then we supplied $\mathrm{As}_{4}$ to crystallize the droplets into GaAs dots at $200^{\circ} \mathrm{C}$, followed by annealing at $500{ }^{\circ} \mathrm{C}$. Several microscope observations which include in vacuo scanning tunneling microscopy and atomic force microscopy revealed the formation of dots with a truncated cone shape whose average radius and height were 16 and $1.4 \mathrm{~nm}$, respectively. Figure 1(b) shows the atomic force microscope image of an investigated dot, which exhibits no lateral elongation. This is in stark contrast to dots grown on (100) surfaces, which exhibit significant elongation along [1-10]..$^{32,33}$ The GaAs dots were capped with an $\mathrm{Al}_{0.3} \mathrm{Ga}_{0.7} \mathrm{As}$ barrier.

As an excitation source we used a pulsed semiconductor laser with a high repetition frequency of $200 \mathrm{MHz}{ }^{34-37}$ We simultaneously counted three photon channels, ${ }^{38}$ i.e., $X X$ photons projected onto a given polarization state, $X$ photons projected onto another polarization state (such as $|R\rangle$ ), and its orthogonal complement (such as $|L\rangle$ ). The use of three detectors enabled us to eliminate the influence of excitation fluctuations on coincidence visibility. ${ }^{39}$ The number of coincidence was analyzed with a time-to-digital converter. The typical integration time was $10 \mathrm{~min}$ for each polarization condition. All the experiments were performed at $9 \mathrm{~K}$.

Correlation measurements: Entanglement fidelity. Figure 1(c) shows the photoluminescence (PL) spectrum of an isolated dot. It consists of four main lines, which are identified as being from the high-energy side, neutral excitons $(X)$, positively charged excitons $\left(X^{+}\right)$, neutral biexcitons $(X X)$, and negatively charged excitons $\left(X^{-}\right) \cdot{ }^{40}$ For performing the correlation measurements, we select as-grown dots without a detectable FSS from the sample. Polarized PL was analyzed with a spectral resolution comparable to the radiative width, (a)

(b)

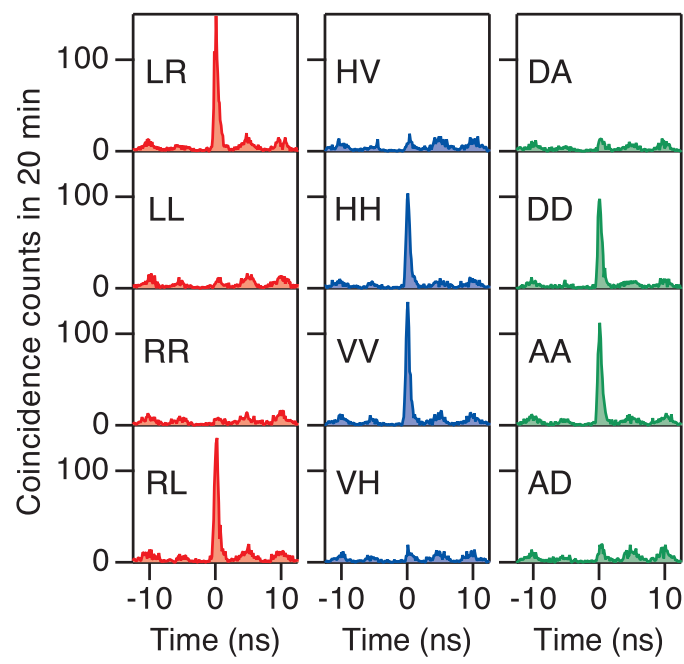

FIG. 2. (Color online) Coincidence histograms between the $X X$ and $X$ photons for different polarization combinations. The signal at positive times is counted for the detection of an $X X$ photon followed by that of an $X$ photon. The two-photon projection settings (such as $L R$ ) are indicated by the first letter for $X X$ photons and the second letter for $X$ photons. They are plotted with a time bin of $128 \mathrm{ps}$.

which is expected to be $1.2 \mu \mathrm{eV}$ [560 ps in terms of lifetime; see Fig. 4(a)]. Small but nonzero FSS values are confirmed for most of the dots, and they are distributed around a mean value of $10 \pm 5 \mu \mathrm{eV}$. This is noticeably smaller than both the typical values for Stranski-Krastanov grown dots, and those for droplet epitaxial GaAs dots grown on (100). ${ }^{32}$ In the investigated sample $5 \%$ of the dots show no detectable FSS. We have measured the photon correlations in more than ten selected dots and they all exhibit entanglement.

Figure 2 shows the results of photon correlation measurements in a typical dot. See Fig. 1 in the Supplemental Material $^{39}$ for a spectral characterization in this dot. $L$, $R, H$, and $V$ indicate projections along the left-handed circular, right-handed circular, linear laboratory horizontal, and vertical polarizations, respectively. $D$ is linear diagonal with a polarization axis tilted by $45^{\circ}$ from $H$, and $A$ is antidiagonal where $A \perp D$. The top panel in Fig. 2(a) shows a coincidence histogram for $L$-polarized $X X$ photons and $R$-polarized $X$ photons (denoted by $L R$ ). The presence of a central peak confirms a radiative cascade. The $X X$ and $X$ photons are clearly correlated, resulting in a higher probability than that for detecting uncorrelated photons. The central peak disappears for a polarization combination of $L L$ (second panel). Thus, the probability of observing both $X X$ and $X$ photons in $L$ is close to zero. The same anticorrelation is confirmed for $R R$ (third panel), but a positive correlation is recovered for $R L$ (bottom panel). These results imply that the two-photon polarization state can be approximated by one of the Bell (maximally entangled) states,

$$
|\Psi\rangle=\frac{|L R\rangle+|R L\rangle}{\sqrt{2}} .
$$

A key criterion for entanglement is the presence of a correlation independent of the chosen polarization basis. 
Figure 2(b) shows coincidence histograms for rectilinear polarizations. A positive correlation appears for parallel polarizations ( $H H, V V)$, while it disappears for perpendicular polarizations $(H V, V H)$. These results agree with the expression of the Bell state of Eq. (1) in a linear polarization basis,

We define the correlation visibility $C=\mid\left(n_{\|}-n_{\perp}\right) /$ $\left(n_{\|}+n_{\perp}\right) \mid$, where $n_{\|}$is the number of coincidences normalized with the two-photon flux for a co-polarized basis, and $n_{\perp}$ is that for a cross-polarized basis (see the Supplemental Material for the normalization procedure). ${ }^{39}$ An ideal source is expected to show $C=1$ for any orthogonal basis set. Our results show that $C=0.87 \pm 0.03$ for $R / L$ and $C=0.78 \pm 0.03$ $(0.77 \pm 0.03)$ for $H / V(D / A)$. The visibility for linear polarizations is found to be approximately independent of the polarization direction, which demonstrates the isotropic characteristic of our source [Fig. 2(a) in the Supplemental Material]. ${ }^{39}$ The higher $C$ value for the circular basis than for the linear bases originates from the hyperfine interaction of the exciton with nuclear spins (see the Supplemental Material ${ }^{39}$ for discussion). The entanglement fidelity is defined as the projection amplitude of a measured polarization state on a target Bell state, which is given by $f=\left(1+C_{R / L}+C_{H / V}+\right.$ $\left.C_{D / A}\right) / 4 .{ }^{20}$ Our results reveal that $f=0.86( \pm 0.02)$, which is much larger than the classical limit of 0.5 , and rates among the best reported in previous studies on dot-based photon sources. ${ }^{12,15-18}$

Experimental violation of Bell's inequality. Entangled photon pair emission from quantum dots (QDs) is an important milestone, but the most powerful applications of entanglement are linked to nonlocality, which is only assured by the violation of Bell's inequality. ${ }^{1}$ To verify this experimentally, we have performed additional photon correlation experiments in nonorthogonal polarization bases. Figure 3(a) shows normalized coincidence counts as a function of the polarization angle of $X\left(\theta_{X}\right)$ at four different angle settings for $X X$ polarization $\left(\theta_{X X}\right)$. Note that we define the angle of $\theta$ as the polar angle of a polarization state that moves in the $R L H V$ plane of the Poincare sphere $\left(\theta=0\right.$ for $R$ and $\theta=90^{\circ}$ for $H$ ). It was experimentally controlled by the application of phase retardance to each beam using liquid crystals. The azimuthangle dependence was also measured and shown in Fig. 2(b) in the Supplemental Material. ${ }^{39}$ Sinusoidal oscillations in the coincidence counts provide evidence of quantum interference, distinct from classical correlation. The maximum violation of Bell's inequality in the Clauser-Horne-Shimony-Holt form ${ }^{41}$ is expected to appear for polarization correlations with $\theta_{X X}=0^{\circ}$, $90^{\circ}, 180^{\circ}, 270^{\circ}$ and $\theta_{X}=45^{\circ}, 135^{\circ}, 225^{\circ}$, and $315^{\circ}$. We measure the coincidence counts for these settings, and estimate the $S$ parameter to be $2.33 \pm 0.06>2$. It clearly violates Bell's inequality by more than five times the standard deviation, which is definite proof of the nonlocality of the measured photons. The importance of nonlocality of entanglement is seen in the Ekert protocol. ${ }^{3}$ If an eavesdropper measures either state of an entangled pair, it becomes local reality and nonlocality vanishes. This is why Bell's inequality can serve as a secure test against eavesdropping. Other applications such as quantum teleportation and entanglement swapping also rely on nonlocality. Consequently, our device is a QD source which can be used for real applications without applying temporal or spectral filtering techniques. (a)
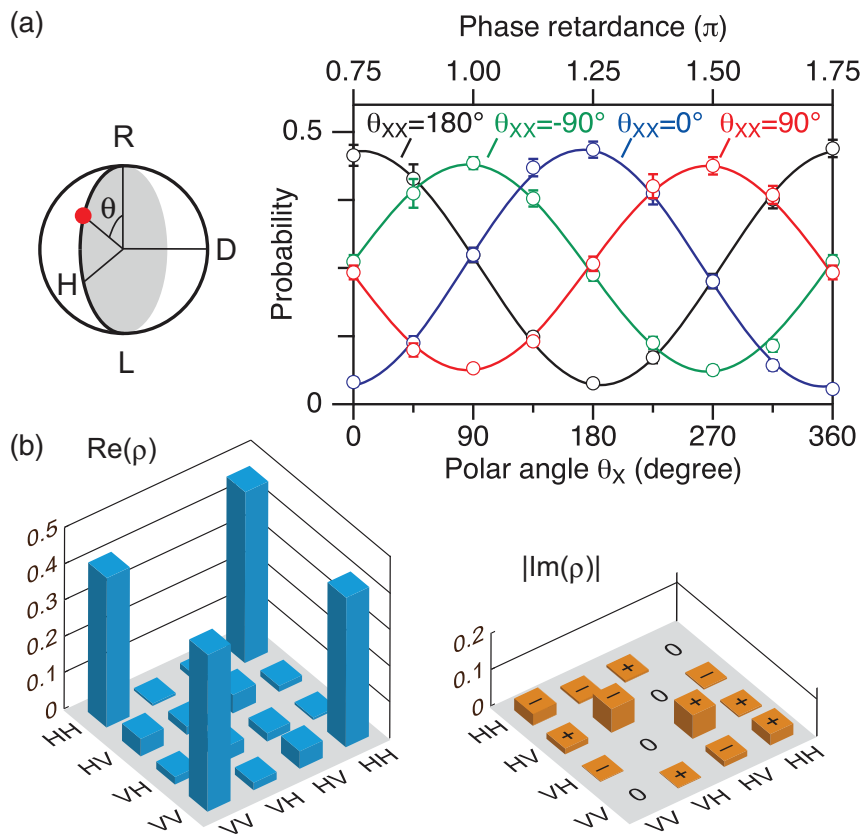

FIG. 3. (Color online) (a) Normalized coincidence counts as a function of the $X$ polarization angle $\left(\theta_{X}\right)$ for four different values of the $X X$ polarization $\left(\theta_{X X}\right)$. The error bars include only Poissonian noise. The sinusoidal fits are also shown by lines. (b) Tomographic representation of the measured two-photon state. The density matrix is reconstructed using coincidence counts for 36 projection bases. The absolute values are plotted for the imaginary part of the matrix, and their signs are shown in the top of each element.

Figure 3(b) shows the reconstructed density matrix of the two-photon state using the correlation measurement results of 36 projection sets $(X, X X \in\{R, L, H, V, D, A\})$ with the aid of a maximum-likelihood technique. ${ }^{42}$ The presence of four real values at the corner of the matrix, with negligible values for the others, demonstrates the superior characteristics of our source. The matrix has a partial transpose with the minimum eigenvalue of $-0.36<0$, which clearly satisfies the Peres criterion of entanglement, which assures quantum inseparability. ${ }^{43}$ The density matrix allows us to evaluate the degree of coherence and the degree of mixedness of the measured state in terms of the tangle $(T)$ and the linear entropy $\left(S_{L}\right)$, respectively. From $T$ we derive one of the most basic measure of the entanglement of formation $\left(E_{F}\right){ }^{44}$ Our results reveal that $\left(T, S_{L}, E_{F}\right)=(0.53,0.32,0.63)$. Note that we achieve these values even without the postselection (or any local operation) of the photons.

The small but apparent deviation in the measured photons from the ideal Bell pairs [Eq. (1)] is due to the depolarization of the exciton state. Figure 4(a) shows the time-resolved PL of the $X$ line after polarized quasiresonant excitation. Note that we study the same dot as that used in the correlation measurement. The PL decay shows a single exponent with a lifetime of $\Gamma_{1}^{-1}=560 \mathrm{ps}$, which is fully consistent with the exciton dipole moment determined by a Rabi oscillation measurement. ${ }^{45}$ Figure 4(b) shows the circular polarization degree, which decays with $\Gamma_{\mathrm{s}}^{-1}=1.5 \mathrm{~ns}$. The fact that $\Gamma_{\mathrm{s}} \ll \Gamma_{1}$ supports the view that polarization memory is well conserved until recombination. Nevertheless, a finite value for $\Gamma_{\mathrm{s}}$ gives rise 

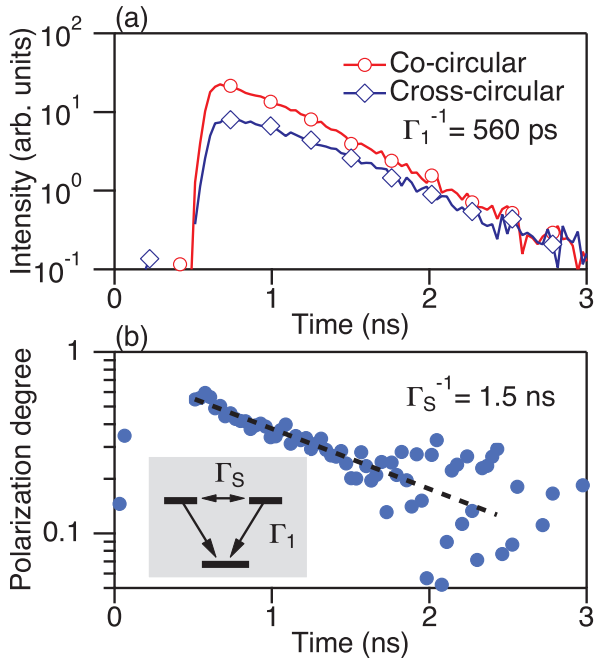

FIG. 4. (Color online) (a) The decay of circularly polarized PL signals for the $X$ line after short-pulsed and quasiresonant excitation. Here we study the same dot as that used in the correlation measurement. (b) The degree of circular polarization, defined as $[I(\sigma)-I(\bar{\sigma})] /[I(\sigma)+I(\bar{\sigma})]$, where $I(\sigma)$ and $I(\bar{\sigma})$ are the cocircular and cross-circular intensities, respectively. The dashed line is an exponential fit to data, with an estimated decay time of $\Gamma_{\mathrm{s}}^{-1}=1.5$ $n s$. The inset shows the energy diagram of the exciton state. In the experiment, we used a short-pulsed parametric oscillator that emitted 4 ps pulses with a wavelength shifted by an optical phonon energy of $37 \mathrm{meV}$ from the $X$ line. The excitation polarization was set as circular, and temporally modulated to maintain an equilibrium nuclear environment. Polarized PL was detected by a fast-response photomultiplier tube with a response time of $40 \mathrm{ps}$.

to a finite probability of observing depolarized photons. We can estimate the correlation visibility of photon pairs to be $\Gamma_{1} /\left(\Gamma_{1}+\Gamma_{\mathrm{s}}\right) \approx 0.7$, which is in fairly good agreement with the observed $C$ value. These findings indicate that our source is neither affected by incoherent noise associated with carrier recapturing ${ }^{17,20}$ nor light emission from other luminescent centers than the dot. The degree of entanglement is thus purely limited by the scattering of excitons. We ascribe the exciton depolarization to random charge and nuclear spin fluctuations in and near the dot. The slowly varying environment represents a remaining source of asymmetry that limits the degree of quantum interference even in a solid-state photon source based on symmetric dots, as discussed in the Supplemental Material. ${ }^{39}$

In summary, we have demonstrated the generation of entangled photon pairs using a strain-free GaAs dot as a symmetric artificial-atom cascade on (111) $A$ surfaces. A clear violation of Bell's inequality is observed in correlation measurements that do not rely on postselection through filtering or tuning. Using our source in quantum cryptography applications would allow safe key distribution not based on a mathematical difficulty but on a fundamental physical law that protects the system, namely, the completeness of quantum mechanics. ${ }^{3}$ The purity of our source also paves the way for using droplet dot-based emitters to investigate the exact connection between the security of quantum cryptography and tests of quantum nonlocality ${ }^{24}$ in the solid state. We clarified the impact of the remaining exciton depolarization on the degree of entanglement in the emitted pairs. The influence of depolarization could be efficiently suppressed in the future by using the Purcell enhancement of the radiative rate $^{17}$ or time-domain filtering with fastresponse detectors. ${ }^{20}$ As droplet epitaxy is not strain driven, a large variety of dot-barrier material combinations can be grown. This opens up the possibility of extending the current emission wavelengths around $700 \mathrm{~nm}$ (that yields the highest photon counting efficiency in Si-based detectors) towards the wavelength regions suitable for long-distance fiber-based telecommunication.

Note added. Recently, entangled photon emission from dots grown on patterned (111)B substrates has been reported. ${ }^{46}$

This work was supported in part by a Grant-in-Aid from JSPS, ITN SpinOptronics, and ERC StG OptoDNPcontrol (B.U.).
${ }^{1}$ J. S. Bell, Physics 1, 195 (1964).

${ }^{2}$ A. Einstein, B. Podolsky, and N. Rosen, Phys. Rev. 47, 777 (1935).

${ }^{3}$ A. K. Ekert, Phys. Rev. Lett. 67, 661 (1991).

${ }^{4}$ T. Jennewein, C. Simon, G. Weihs, H. Weinfurter, and A. Zeilinger, Phys. Rev. Lett. 84, 4729 (2000).

${ }^{5}$ D. S. Naik, C. G. Peterson, A. G. White, A. J. Berglund, and P. G. Kwiat, Phys. Rev. Lett. 84, 4733 (2000).

${ }^{6}$ O. Benson, C. Santori, M. Pelton, and Y. Yamamoto, Phys. Rev. Lett. 84, 2513 (2000).

${ }^{7}$ A. Aspect, P. Grangier, and G. Roger, Phys. Rev. Lett. 47, 460 (1981).

${ }^{8}$ A. Aspect, P. Grangier, and G. Roger, Phys. Rev. Lett. 49, 91 (1982).

${ }^{9}$ D. Gammon, E. S. Snow, B. V. Shanabrook, D. S. Katzer, and D. Park, Science 273, 87 (1996).

${ }^{10}$ M. Bayer, A. Kuther, A. Forchel, A. Gorbunov, V. B. Timofeev, F. Schäfer, J. P. Reithmaier, T. L. Reinecke, and S. N. Walck, Phys. Rev. Lett. 82, 1748 (1999).
${ }^{11}$ C. Santori, D. Fattal, M. Pelton, G. S. Solomon, and Y. Yamamoto, Phys. Rev. B 66, 045308 (2002).

${ }^{12}$ R. M. Stevenson, R. J. Young, P. Atkinson, K. Cooper, D. A. Ritchie, and A. J. Shields, Nature (London) 439, 179 (2006).

${ }^{13}$ R. J. Young, R. M. Stevenson, P. Atkinson, K. Cooper, D. A. Ritchie, and A. J. Shields, New J. Phys. 8, 29 (2006).

${ }^{14}$ N. Akopian, N. H. Lindner, E. Poem, Y. Berlatzky, J. Avron, D. Gershoni, B. D. Gerardot, and P. M. Petroff, Phys. Rev. Lett. 96, 130501 (2006).

${ }^{15}$ R. Hafenbrak, S. M. Ulrich, P. Michler, L. Wang, A. Rastelli, and O. G. Schmidt, New J. Phys. 9, 315 (2007).

${ }^{16}$ A. Muller, W. Fang, J. Lawall, and G. S. Solomon, Phys. Rev. Lett. 103, 217402 (2009).

${ }^{17}$ A. Dousse, J. Suffczynski, A. Beveratos, O. Krebs, A. Lemaître, I. Sagnes, J. Bloch, P. Voisin, and P. Senellart, Nature (London) 466, 217 (2010). 
${ }^{18}$ M. Ghali, K. Ohtani, Y. Ohno, and H. Ohno, Nat. Commun. 3, 661 (2012)

${ }^{19}$ R. Trotta, E. Zallo, C. Ortix, P. Atkinson, J. D. Plumhof, J. van den Brink, A. Rastelli, and O. G. Schmidt, Phys. Rev. Lett. 109, 147401 (2012).

${ }^{20}$ R. J. Young, R. M. Stevenson, A. J. Hudson, C. A. Nicoll, D. A. Ritchie, and A. J. Shields, Phys. Rev. Lett. 102, 030406 (2009).

${ }^{21}$ T. Mano, M. Abbarchi, T. Kuroda, B. McSkimming, A. Ohtake, K. Mitsuishi, and K. Sakoda, Appl. Phys. Express 3, 065203 (2010).

${ }^{22}$ G. Sallen, B. Urbaszek, M. M. Glazov, E. L. Ivchenko, T. Kuroda, T. Mano, S. Kunz, M. Abbarchi, K. Sakoda, D. Lagarde, A. Balocchi, X. Marie, and T. Amand, Phys. Rev. Lett. 107, 166604 (2011).

${ }^{23}$ M. V. Durnev, M. M. Glazov, E. L. Ivchenko, M. Jo, T. Mano, T. Kuroda, K. Sakoda, S. Kunz, G. Sallen, L. Bouet, X. Marie, D. Lagarde, T. Amand, and B. Urbaszek, Phys. Rev. B 87, 085315 (2013).

${ }^{24}$ N. Gisin, G. Ribordy, W. Tittel, and H. Zbinden, Rev. Mod. Phys. 74, 145 (2002).

${ }^{25}$ R. Seguin, A. Schliwa, S. Rodt, K. Pötschke, U. W. Pohl, and D. Bimberg, Phys. Rev. Lett. 95, 257402 (2005).

${ }^{26}$ R. Singh and G. Bester, Phys. Rev. Lett. 103, 063601 (2009).

${ }^{27}$ A. Schliwa, M. Winkelnkemper, A. Lochmann, E. Stock, and D. Bimberg, Phys. Rev. B 80, 161307 (2009).

${ }^{28}$ Y. Sugiyama, Y. Sakuma, S. Muto, and N. Yokoyama, Appl. Phys. Lett. 67, 256 (1995).

${ }^{29}$ A. Mohan, M. Felici, P. Gallo, B. Dwir, A. Rudra, J. Faist, and E. Kapon, Nat. Photonics 4, 302 (2010); 6, 793 (2012).

${ }^{30}$ E. Stock, T. Warming, I. Ostapenko, S. Rodt, A. Schliwa, J. A. Töfflinger, A. Lochmann, A. I. Toropov, S. A. Moshchenko, D. V. Dmitriev, V. A. Haisler, and D. Bimberg, Appl. Phys. Lett. 96, 093112 (2010).

${ }^{31}$ R. J. Young, R. M. Stevenson, A. J. Shields, P. Atkinson, K. Cooper, D. A. Ritchie, K. M. Groom, A. I. Tartakovskii, and M. S. Skolnick, Phys. Rev. B 72, 113305 (2005).

${ }^{32}$ M. Abbarchi, C. A. Mastrandrea, T. Kuroda, T. Mano, K. Sakoda, N. Koguchi, S. Sanguinetti, A. Vinattieri, and M. Gurioli, Phys. Rev. B 78, 125321 (2008).

${ }^{33}$ Y.-H. Liao, C.-C. Liao, C.-H. Ku, Y.-C. Chang, S.-J. Cheng, M. Jo, T. Kuroda, T. Mano, M. Abbarchi, and K. Sakoda, Phys. Rev. B 86, 115323 (2012).
${ }^{34}$ The laser pulse width was $80 \mathrm{ps}$, and the wavelength $640 \mathrm{~nm}$. The high repetition pumping allowed us to measure the correlation profile with high visibility (Refs. 35 and 36). In this condition, photocarriers were pumped to the barrier continuum, and then relaxed to dots before recombination. Linear excitation polarization was employed to avoid the effects of nuclear polarization (Ref. 37).

${ }^{35}$ G. Oohata, R. Shimizu, and K. Edamatsu, Phys. Rev. Lett. 98, 140503 (2007).

${ }^{36}$ T. Kuroda, T. Belhadj, M. Abbarchi, C. Mastrandrea, M. Gurioli, T. Mano, N. Ikeda, Y. Sugimoto, K. Asakawa, N. Koguchi, K. Sakoda, B. Urbaszek, T. Amand, and X. Marie, Phys. Rev. B 79, 035330 (2009).

${ }^{37}$ T. Belhadj, T. Kuroda, C.-M. Simon, T. Amand, T. Mano, K. Sakoda, N. Koguchi, X. Marie, and B. Urbaszek, Phys. Rev. B 78, 205325 (2008).

${ }^{38}$ The photoluminescence emission from a single dot was collected by an objective lens with a numerical aperture of 0.75 , and split into two beams with different spectral components, one comprising the $X$ line and the other comprising the $X X$ line with a bandwidth of $200 \mu \mathrm{eV}$. Each beam was coupled to a polarization analyzer and detected by an avalanche photodiode. The typical count rate was 30000 counts s${ }^{-1}$ for $X$ photons, and 6000 counts s ${ }^{-1}$ for $X X$ photons, when the polarization analyzer was removed.

${ }^{39}$ See Supplemental Material at http://link.aps.org/supplemental/ 10.1103/PhysRevB.88.041306 for a spectral characterization in our dot (Fig. 1), photon correlation results using linear polarization bases (Fig. 2), discussion on the source of entanglement degradation, and description of normalization procedure used in the correlation analysis.

${ }^{40}$ M. Abbarchi, T. Kuroda, T. Mano, K. Sakoda, C. A. Mastrandrea, A. Vinattieri, M. Gurioli, and T. Tsuchiya, Phys. Rev. B 82, 201301 (2010).

${ }^{41}$ J. F. Clauser, M. A. Horne, A. Shimony, and R. A. Holt, Phys. Rev. Lett. 23, 880 (1969).

${ }^{42}$ D. F. V. James, P. G. Kwiat, W. J. Munro, and A. G. White, Phys. Rev. A 64, 052312 (2001).

${ }^{43}$ A. Peres, Phys. Rev. Lett. 77, 1413 (1996).

${ }^{44}$ W. K. Wootters, Phys. Rev. Lett. 80, 2245 (1998).

${ }^{45}$ K. Kuroda, T. Kuroda, K. Watanabe, T. Mano, K. Sakoda, G. Kido, and N. Koguchi, Appl. Phys. Lett. 90, 051909 (2007).

${ }^{46}$ G. Juska, V. Dimastrodonato, L. O. Mereni, A. Gocalinska, and E. Pelucchi, Nat. Photonics 7, 527 (2013). 